\title{
Una Escala de Evaluación Familiar Eco-Sistémica para Programas Sociales: Confiabilidad y Validez de la NCFAS en Población de Alto Riesgo Psicosocial
}

\author{
An Eco-Systemic Family Assessment Scale for Social \\ Programs: Reliability and Validity of NCFAS in a High \\ Psychosocial Risk Population
}

\author{
Edgar Valencia \\ Pontificia Universidad Católica de Chile
}

\author{
Esteban Gómez \\ Protectora de la Infancia
}

\begin{abstract}
Se presenta evidencia sobre la consistencia interna y validez de constructo de la versión en español de la Escala de Evaluación Familiar de Carolina del Norte (NCFAS). El estudio utilizó los registros de información de 528 participantes de 8 programas chilenos de intervención familiar para la prevención del maltrato y negligencia infantil. Los resultados indican que la escala tiene una consistencia interna apropiada, mostrando un comportamiento similar al de la versión original de la escala. El análisis factorial exploratorio replicó parcialmente las dimensiones teóricas del instrumento. Se sugiere distribuir los ítems de la dimensión Interacciones Familiares e incorporar un nuevo factor llamado Bienestar del Cuidador. Se discute la necesidad de adaptar la NCFAS al contexto latinoamericano en futuras investigaciones.
\end{abstract}

Palabras clave: escala de evaluación familiar, NCFAS, propiedades psicométricas, familia multiproblemática, programas de intervención familiar.

\begin{abstract}
Evidence about the internal consistency and construct validity of the Spanish version of the North Carolina Family Assessment Scale (NCFAS) is presented. The study used records of 8 Chilean family preservation programs for prevention of child abuse and neglect $(N=528)$. The results indicate that the scale has appropriate internal consistency, showing a behavior similar to the one of the original version of the scale. The exploratory factor analysis partially supports the theoretical domains of the assessment tool. Distributing the items of the Family Interactions domain and including a new factor called Caregiver's Well-being is suggested. The necessity of adapting the NCFAS to a LatinAmerican context in future research is discussed.
\end{abstract}

Keywords: family assessment scale, NCFAS, psychometric properties, multi-problem family, family preservation programs.

\section{Introducción}

En los últimos años las organizaciones sociales en Chile y Latinoamérica han iniciado un proceso de profesionalización y tecnificación de sus servicios y programas. Sin embargo, aún se encuentran lejos de ofrecer una imagen consolidada de eficiencia y eficacia (Rodríguez \& Ríos, 2007). Uno de los mayores desafíos continúa siendo la generación de sistemas de evaluación con- fiables, válidos y multi-dimensionales, que permitan reflejar la complejidad de los fenómenos sociales que se pretende intervenir, por ejemplo, integrando diversas fuentes de información (Matus, 2007). Parte de las dificultades es la carencia de instrumentos de evaluación con propiedades psicométricas adecuadas, pertinentes y de utilidad práctica en el ejercicio cotidiano de los programas sociales y que, al mismo tiempo, favorezcan y faciliten la labor de los investigadores.

Edgar Valencia Acuña, MIDE UC, Pontificia Universidad Católica de Chile, Santiago, Chile.

Esteban Gómez Muzzio, Protectora de la Infancia, Santiago, Chile.

La correspondencia relativa a este artículo debe ser dirigida a Esteban Gómez, Unidad de Diseño y Evaluación de Programas, Protectora de la Infancia, Avda. Concha y Toro 1898, Santiago, Chile. E-mail: egomez@protectora.cl Los autores agradecen a Priscilla Martens, Directora Ejecutiva del National Family Preservation Network en Estados Unidos, por facilitar la escala NCFAS y su traducción al español. 
Uno de los campos que evidencia con urgencia esta necesidad es la prevención y protección frente a las diversas formas de maltrato infantil y negligencia parental que enfrentan millones de niños/as cada año (Larraín \& Bascuñán, 2008; SantanaTavira, Sánchez-Ahedo \& Herrera-Basto, 1998). Estas situaciones se dan con mayor frecuencia en familias multiproblemáticas, en que coexisten numerosos factores de vulnerabilidad, primando en las explicaciones etiológicas actuales modelos acumulativos y complejos de factores de riesgo, por sobre modelos lineales o unidimensionales (Appelyard, Egeland, van Dulmen \& Sroufe, 2005; Connell-Carrick, 2003; Gómez \& Haz, 2008; Herrenkohl \& Herrenkohl, 2007). Estas temáticas demandan la intervención de equipos multidisciplinarios y habitualmente convocan a diversos sistemas, como instituciones judiciales, organizaciones sociales, el sistema escolar y de salud, entre otros (Gómez, Muñoz \& Haz, 2007).

Para avanzar se requieren herramientas de evaluación que capturen la estructura ecológica del funcionamiento familiar (Belsky, 1993; Stockhammer, Salzinger, Feldman, Mojica \& Primavera, 2001), permitiendo ponderar fortalezas y déficit en diversas dimensiones (por ejemplo, conocimientos, actitudes y conductas) y facilitando dar respuesta simultánea a las preocupaciones de los sistemas de bienestar y protección infantil, los servicios de salud mental y los sistemas de justicia familiar y/o juvenil, en cada país. Además, dada la naturaleza de los programas psicosociales, es fundamental que requieran un mínimo de entrenamiento para usarse, tomen poco tiempo de administración y puedan detectar pequeños cambios en el funcionamiento familiar durante intervenciones breves (Johnson et al., 2006). Finalmente, un aspecto gravitante es que cumplan con altos estándares en validez y confiabilidad (Carretero-Dios \& Pérez, 2007).

En Chile se dispone de escasas herramientas para evaluar familias. Una notable excepción es la Escala del Estilo de Funcionamiento Familiar que, mediante un cuestionario de 26 afirmaciones (a las cuales responde la familia en un continuo de cinco puntos), permite identificar con fines psicoterapéuticos: la identidad familiar, información compartida y recursos de afrontamiento; mostrando propiedades psicométricas adecuadas (Larraín, Zegers, Diez, Trapp \& Polaino-Lorente, 2003). Sin embargo, no se cuenta con instrumentos que evalúen el funcionamiento familiar desde la perspectiva de los profesionales ni que tengan como finalidad su uso en programas psicosociales.

El presente artículo pretende contribuir en esta dirección, difundiendo la Escala de Evaluación Familiar de Carolina del Norte (North Carolina Family Assessment Scale, NCFAS) y presentando antecedentes sobre sus propiedades psicométricas relacionadas con la consistencia interna y validez de constructo del instrumento.

\section{Antecedentes}

La NCFAS es una escala de evaluación familiar integral desarrollada a mediados de 1990 por un grupo de trabajo ligado al Sistema de Bienestar Infantil de Carolina del Norte (agencias de protección gubernamental, fundaciones/organizaciones sociales y universidad), liderado por los investigadores Raymond Kirk y Kellie Reed-Ashcraft y apoyados por la organización National Family Preservation Network (NFPN), para ser usada en programas de preservación familiar orientados a la prevención secundaria del maltrato infantil, negligencia y otros factores de riesgo que puedan colocar a un niño en situación de grave vulneración.

Basándose en la teoría ecológica del desarrollo humano (Bronfenbrenner, 1986, 1987), la NCFAS busca evaluar el constructo funcionamiento familiar (Johnson et al., 2006; Larraín et al., 2003), mediante la inclusión de una serie de indicadores que han mostrado tener relevancia empírica, agrupándolos en dimensiones que pueden homologarse a los niveles microsistémico, mesosistémico y exosistémico del modelo ecológico (Reed-Ashcraft, Kirk \& Fraser, 2001).

La NCFAS ha sido reconocida como un valioso instrumento con aplicaciones prácticas y académicas, habiendo sido usada en la evaluación de programas de visita domiciliaria con padres primerizos (De la Rosa, Perry, Dalton \& Johnson, 2005; 
De la Rosa, Perry \& Johnson, 2009), programas de prevención temprana del maltrato infantil (Fernandez, 2007), servicios intensivos de preservación familiar (Kirk \& Griffith, 2007), programas de intervención culturalmente sensibles (Richardson, 2008), una intervención judicial piloto en violencia doméstica (Coll \& Stewart, 2006) $\mathrm{y}$ en el estudio de factores de riesgo para la re-hospitalización psiquiátrica (Walker, Minor-Schork, Bloch \& Esinhart, 1996). En sus distintas versiones, la NCFAS se aplica actualmente en cerca de 450 agencias y organizaciones en Estados Unidos, Canadá y Australia (NFPN, 2009).

El proceso completo de desarrollo del instrumento se encuentra disponible en la literatura de trabajo social (Kirk, Kim \& Griffith, 2005; Reed, 1998; Reed-Ashcraft et al., 2001). La escala ha sido revisada y perfeccionada, contando con 36 ítems en su versión actual 2.0: cinco ítems globales y 31 ítems específicos, distribuidos según se especifica en la Tabla 1 (Kirk, Reed-Ashcraft \& NFPN, 2007). Las cinco dimensiones de la NCFAS son: a) Entorno, que se refiere al contexto de desarrollo del niño y pondera condiciones de salubridad, seguridad y es- tabilidad familiar cotidiana; b) Competencias Parentales, referida a conocimientos, conductas y características de los padres/ madres o cuidadores para criar adecuadamente; c) Interacciones Familiares, que evalúa la relación entre los distintos miembros de la familia; d) Seguridad Familiar, que identifica situaciones que amenacen la integridad física y emocional de sus miembros, como maltrato infantil o violencia de pareja; y e) Bienestar del Niño, que permite ponderar el desempeño actual del niño/a o adolescente en diversos ámbitos de su vida, como la escuela, relación con pares o salud mental.

\section{Uso de la NCFAS}

En la práctica, el uso de la escala es bastante sencillo. Generalmente, en todo programa de intervención familiar se realizan procedimientos de evaluación inicial, como entrevistas, visitas domiciliarias y cuestionarios (Fernández, 2007; Gómez \& Haz, 2008). Dicha información permite a los profesionales formarse un juicio sobre diversas características del funcionamiento familiar actual. Lo que aporta la NCFAS es

Tabla 1

Dominios e Ítems de la Escala de Evaluación Familiar de Carolina del Norte (NCFAS) en Español

\begin{tabular}{|c|c|c|c|}
\hline Dominio & $\begin{array}{l}\mathrm{N}^{\mathrm{o}} \text { Total } \\
\text { de Ítems }\end{array}$ & $\begin{array}{l}\text { Ítem de } \\
\text { Ponderación Global } \\
\text { del Dominio }\end{array}$ & Ítems Específicos que Conforman el Dominio \\
\hline Entorno & 10 & entorno & $\begin{array}{l}\text { estabilidad de la vivienda; seguridad en la comunidad; habi- } \\
\text { tabilidad de la vivienda; ingreso/empleo; manejo financiero; } \\
\text { comida y nutrición; higiene personal; transporte; entorno de } \\
\text { aprendizaje }\end{array}$ \\
\hline $\begin{array}{l}\text { Competencias } \\
\text { Parentales }\end{array}$ & 7 & $\begin{array}{l}\text { competencias } \\
\text { parentales }\end{array}$ & $\begin{array}{l}\text { supervisión del niño/s; prácticas disciplinarias; entrega de } \\
\text { oportunidades de desarrollo/crecimiento; salud mental del } \\
\text { progenitor/es/cuidador/es; salud física del progenitor/es/cui- } \\
\text { dador/es; uso de drogas/alcohol del progenitor/es/cuidador/es }\end{array}$ \\
\hline $\begin{array}{l}\text { Interacciones } \\
\text { Familiares }\end{array}$ & 5 & $\begin{array}{l}\text { interacciones } \\
\text { familiares }\end{array}$ & $\begin{array}{l}\text { apego con el niño/s; expectativas sobre el niño/s; apoyo } \\
\text { mutuo dentro de la familia; relación entre los progenitores/ } \\
\text { cuidadores }\end{array}$ \\
\hline $\begin{array}{l}\text { Seguridad } \\
\text { Familiar }\end{array}$ & 6 & seguridad familiar & $\begin{array}{l}\text { abuso físico en el niño/s; abuso sexual en el niño/s; abuso } \\
\text { emocional en el niño/s; negligencia en el niño/s; violencia } \\
\text { doméstica entre los progenitores/cuidadores }\end{array}$ \\
\hline $\begin{array}{l}\text { Bienestar del } \\
\text { Niño }\end{array}$ & 8 & bienestar del niño & $\begin{array}{l}\text { salud mental del niño/s; comportamiento del niño/s; } \\
\text { desempeño escolar; relación con el progenitor/es/cuidador/ } \\
\text { es; relación con hermano/s; relación con pares; cooperación/ } \\
\text { motivación a permanecer en la familia }\end{array}$ \\
\hline
\end{tabular}


un ordenamiento de esa información, requiriendo que el profesional asigne puntajes en las cinco dimensiones y sus ítems respectivos en base a criterios consignados en un manual.

La NCFAS consiste en un formulario (versión en papel o electrónica) de cinco hojas, una por cada dimensión; primero la dimensión global y luego sus ítems correspondientes (ver ejemplo en la Figura 1). Las dimensiones y sus ítems permiten evaluar el funcionamiento familiar actual en un continuo de seis puntos: problema serio (-3), problema moderado (-2), problema leve (-1), línea base/adecuado (0), leve fortaleza $(+1)$ y clara fortaleza $(+2)$. Los puntajes se asignan en dos ocasiones: cuando la familia ingresa al programa (Ingreso) y al finalizar la intervención (Cierre). Los puntajes del ingreso sirven para desarrollar metas y planes de intervención, mientras que los del cierre permiten evaluar cambios o retrocesos (pudiendo sugerirse nuevas intervenciones y/o servicios en esas áreas).

Cada ítem cuenta con una definición operacional (o criterio) que describe el indicador para las puntuaciones extremas (-3 y +2) y para la línea base (0), guiando el juicio del profesional. En la Tabla 2 se muestra la definición del ítem "Seguridad en la Comunidad" en sus tres puntuaciones y ejemplos de la puntuación -3 para un ítem por cada dimensión. La puntuación +2 representa el mejor nivel de funcionamiento familiar, siendo un recurso, factor protector o mecanismo de resiliencia en la familia. La puntuación 0 o línea base/adecuado se define como el umbral sobre el cual no hay razones legales, morales o éticas para una intervención pública. No significa necesariamente que todo funcione sin problemas o que la familia no se beneficiaría de servicios adicionales si los aceptara voluntariamente, pero no hay una razón poderosa para que el Estado ejerza una protección o mandato de intervención. A su vez, la puntuación -3 implica un serio deterioro del funcionamiento familiar. Cuando se obtienen sistemáticamente puntuaciones de -3, debe revisarse la necesidad de protección del niño y su eventual colocación fuera del sistema familiar (Kirk et al., 2007). Es importante señalar que los puntajes asignados pueden (y deben) cambiarse si aparece nueva información relevante (por ejemplo, una situación de violencia no pesquisada inicialmente), ya que el objetivo de la NCFAS es reflejar una imagen lo más precisa posible del grado de funcionamiento real de la familia.

National Family Preservation Network

\section{B. Competencias Parentales}

Nota: esta sección se refiere al progenitor(es), si está presente, o al cuidador(es) actual.

\begin{tabular}{|c|c|c|c|c|c|c|c|}
\hline & & $\begin{array}{c}\text { Clara } \\
\text { Fortaleza }\end{array}$ & $\begin{array}{c}\text { Leve } \\
\text { Fortaleza }\end{array}$ & $\begin{array}{l}\text { Línea Base/ } \\
\text { Adecuado }\end{array}$ & $\begin{array}{c}\text { Problema } \\
\text { Leve }\end{array}$ & $\begin{array}{l}\text { Problema } \\
\text { Moderado }\end{array}$ & $\begin{array}{c}\text { Problema } \\
\text { Serio }\end{array}$ \\
\hline \multicolumn{8}{|c|}{ 1. Competencias Parentales en general } \\
\hline Ingreso (I) & & +2 & +1 & 0 & -1 & -2 & -3 \\
\hline Cierre (C) & & +2 & +1 & 0 & -1 & -2 & -3 \\
\hline \multicolumn{8}{|c|}{ 2. Supervisión del Niño(s) } \\
\hline Ingreso (I) & $\mathrm{N} / \mathrm{A}$ & +2 & +1 & 0 & -1 & -2 & -3 \\
\hline Cierre (C) & $\mathrm{N} / \mathrm{A}$ & +2 & +1 & 0 & -1 & -2 & -3 \\
\hline \multicolumn{8}{|c|}{ 3. Prácticas Disciplinarias } \\
\hline Ingreso (I) & $\mathrm{N} / \mathrm{A}$ & +2 & +1 & 0 & -1 & -2 & -3 \\
\hline Cierre (C) & N/A & +2 & +1 & 0 & -1 & -2 & -3 \\
\hline
\end{tabular}

Figura 1. Ejemplo del formulario usado en la escala NCFAS. 
Tabla 2

\section{Ejemplos de Indicadores para Ítems de la Escala NCFAS}

\begin{tabular}{|c|c|c|}
\hline \multicolumn{3}{|c|}{ Dimensión Entorno, Ítem "Seguridad en la comunidad" } \\
\hline+2 & Clara Fortaleza & $\begin{array}{l}\text { Vecindario seguro para los niños. Los cuidadores pueden permitir a los niños jugar } \\
\text { afuera sin miedo. Los vecinos se cuidan mutuamente (por ejemplo, los vecinos } \\
\text { "vigilan"). }\end{array}$ \\
\hline 0 & $\begin{array}{l}\text { Línea } \\
\text { Adecuada }\end{array}$ & $\begin{array}{l}\text { Disturbios menores en el vecindario, no evitan que los miembros de la familia y los } \\
\text { niños pasen tiempo afuera en la comunidad. }\end{array}$ \\
\hline-3 & Problema Serio & $\begin{array}{l}\text { Muchos disturbios, tales como peleas y/o revueltas en el vecindario. El vecindario no } \\
\text { es seguro para que los niños jueguen afuera o para que caminen al bus o al colegio. } \\
\text { Evidencia de violencia, ventanas clausuradas o enrejadas, armas de fuego, uso de } \\
\text { alcohol o drogas y/o tráfico de drogas en el vecindario. Vecinos temerosos de "verse } \\
\text { involucrados". }\end{array}$ \\
\hline
\end{tabular}

Dimensión Competencias Parentales, Ítem "Prácticas Disciplinarias"

\begin{tabular}{ll}
\hline & Falta de disciplina de parte de los cuidadores, o a abuso emocional o físico pasado \\
& o actual referido como "disciplina". La disciplina es excesiva, punitiva, inapropiada \\
-3 & a la edad, inconsistente y/o ausente. Presentan modelos de roles pobres. Los \\
& cuidadores están en desacuerdo sobre las estrategias de parentalidad y envían \\
& mensajes mixtos al niño.
\end{tabular}

Dimensión Interacciones Familiares, Ítem "Apego con el niño/s”

\begin{tabular}{|c|c|c|}
\hline-3 & Problema Serio & $\begin{array}{l}\text { Inhabilidad de los cuidadores para formar una relación cercana con sus niños e } \\
\text { incompetencia para criarlos. Los cuidadores están resentidos, rechazadores o } \\
\text { desvinculados con sus hijos. También se refiere a la falta de respuesta, respuesta } \\
\text { inapropiada o involucramiento excesivo con el niño/a. }\end{array}$ \\
\hline \multicolumn{3}{|c|}{ Dimensión Seguridad Familiar, Ítem “Ausencia/Presencia de Negligencia en el Niño/a” } \\
\hline-3 & Problema Serio & $\begin{array}{l}\text { Incidentes/quejas/pruebas de negligencia infantil por parte de los cuidadores, que } \\
\text { no han sido notificadas o abordadas, o se han resuelto en forma insatisfactoria } \\
\text { Los cuidadores pueden estar activamente negando las pruebas de negligencia } \\
\text { resistiendo activamente la intervención. }\end{array}$ \\
\hline \multicolumn{3}{|c|}{ Dimensión Bienestar del Niño, Ítem "Desempeño Escolar" } \\
\hline-3 & Problema Serio & $\begin{array}{l}\text { Niño teniendo una pobre asistencia a la escuela, un pobre rendimiento académico } \\
\text { y/o muchos problemas conductuales en la escuela. El niño declara odiar la escuela } \\
\text { y/o la evita, enfermándose o escapándose. }\end{array}$ \\
\hline
\end{tabular}

Fuente: Escala de Evaluación Familiar de Carolina del Norte, Escala y Definiciones (v 2.0) (Kirk et al., 2007).

\section{Validez y Confiabilidad de la NCFAS}

Diversos estudios realizados en Estados Unidos han examinado la validez y confiabilidad de la NCFAS. Los primeros estudios, desarrollados a fines de 1990, mostraron una adecuada consistencia interna de sus dimensiones $(N=288)$, obteniéndose valores de alfa de Cronbach entre 0,76 y 0,93 (Reed, 1998; Reed-Ashcraft et al., 2001). Asimismo, se evaluó la validez de constructo de las dimensiones de la NCFAS, utilizando como criterio instrumentos estandarizados como el Child Well-Being Scales, Index of Family Relations y Family Inventory of
Resources for Management. Dicho estudio mostró que las correlaciones $r$ de Pearson entre la NCFAS y estos instrumentos eran estadísticamente significativas $(N=126, p$ $<0,01)$ para cinco de seis relaciones estudiadas, con valores entre 0,26 y 0,71 (ReedAshcraft et al., 2001).

El segundo estudio de validación de la NCFAS versión 2.0 abordó la consistencia interna y la validez concurrente y predictiva (Kirk et al., 2005), obteniéndose alfa de Cronbach entre 0,72 y $0,90(N=1279)$. Sobre la validez predictiva, el estudio mostró que las puntuaciones de los niños al final de la intervención estaban directamente aso- 
ciadas con una mayor probabilidad de permanencia en su familia, siendo un criterio de éxito en la intervención $(N=1279)$, replicándose este hallazgo en seguimientos de hasta un año $(N=487)$. En un estudio reciente (Kirk \& Griffith, 2007) se evaluó la efectividad de programas intensivos de preservación familiar, con familias atendidas entre $2004 \mathrm{y}$ 2006. Los niveles de consistencia interna reportados para las dimensiones de la NCFAS fueron alfa de Cronbach entre 0,77 y 0,94.

Asimismo, se han evaluado otras características de la NCFAS. En un estudio realizado en 2006 por el Centro para la Investigación de Servicios Sociales de la Universidad de California en Berkeley (Johnson et al., 2006) se compararon instrumentos de evaluación familiar que cumplieran con los siguientes requisitos: a) abordaje integral de dominios relevantes de evaluación familiar; b) validez y confiabilidad en distintas etapas de un caso; y c) utilidad práctica en el sistema público de bienestar y protección infantil. Los investigadores seleccionaron 85 instrumentos y los compararon en una serie de criterios, como su facilidad de administración (tiempo, instrucciones, puntuación y claridad de interpretación) y sus propiedades psicométricas. Los investigadores identificaron siete instrumentos que cumplieron con la mayoría de los requisitos; la NCFAS se posicionó en el primer lugar, siendo altamente recomendada por los evaluadores (Johnson et al., 2006).

Si bien la consistencia interna y validez de la NCFAS han sido bien establecidas con población estadounidense (Johnson et al., 2006), este estudio es el primero en el que se explora la versión en español del instrumento y con equipos profesionales de Chile.

\section{Adaptación de la NCFAS al Español}

Como parte de un acuerdo de cooperación técnica entre la NFPN y la Protectora de la Infancia, se realizó en 2007 la traducción del instrumento, junto con los materiales de apoyo y capacitación para sus tres versiones: NCFAS, para programas de apoyo a familias que ponen en riesgo la protección y permanencia de un niño en el hogar; NCFAS-R, para programas que evalúan la reunificación familiar en casos de niños ins- titucionalizados en residencias o familias de acogida; y NCFAS-G, para servicios comunitarios generales de apoyo a la familia (NFPN, 2009). El proceso de traducción al español estuvo a cargo de uno de los autores de este estudio y contó con la validación de un panel de jueces expertos. Participaron de este proceso expertos de NFPN y de la Facultad de Educación y Ciencias de la Familia de la Universidad Finis Terrae de Chile. La versión NCFAS en español consta de cinco documentos: a) Escala y Definiciones; b) Preguntas Frecuentes; c) Desarrollo de Metas y un Plan de Caso; d) Estudio de Caso: la familia García; y e) Presentación en Power Point para capacitación (Kirk et al., 2007).

\section{Método}

\section{Muestra}

En este estudio se utilizaron datos de 591 niños/as y adolescentes, en el contexto de la evaluación diagnóstica de los beneficiarios de Programas de Intervención Breve (en adelante PIB) Viviendo en Familia de la Protectora de la Infancia. Estos programas brindan atención integral a familias con indicadores de alto riesgo de maltrato y/o negligencia infantil. Cada centro recibe subvención del Servicio Nacional de Menores de Chile para 70 a 80 niños, sus familias y comunidades, por un plazo entre 12 y 18 meses. Cuentan con equipos multidisciplinarios compuestos por nueve operadores, quienes llevan a cabo un trabajo clínico, psicosocial y comunitario, orientado a garantizar un ambiente familiar seguro y contenedor para los niños/as y adolescentes, potenciar la preservación de la unidad familiar y promover mecanismos protectores en la comunidad. El PIB "Viviendo en Familia" se fundamenta en un enfoque de intervención eco-sistémico, por lo que la escala NCFAS resulta especialmente apropiada. La Protectora de la Infancia implementa 11 de estos centros, ocho de los cuales formaron parte de este estudio.

El promedio de edad de los niños/as y jóvenes participantes fue 9,4 años $(D E=$ 4,2 ), observándose que un $57,2 \%$ de los casos fueron hombres. Se encontraban cur- 
sando mayoritariamente educación básica (63,8\%), seguido por educación preescolar $(19,5 \%)$, educación media $(10,3 \%)$ y ninguna/sin información $(6,4 \%)$.

Los cuidadores principales $(N=354)$ de los niños/as y adolescentes ingresados fueron típicamente madres biológicas en $78,8 \%$, abuelas en $7,3 \%$ y padres biológicos en $6,8 \%$. Su promedio de edad fue 38,3 años $(D E=9,5)$. El nivel de escolaridad alcanzado fue educación media completa/educación superior en $29,6 \%$, básica completa o media incompleta en $34,1 \%$, básica incompleta en $23,4 \%$, sin escolaridad en $3,7 \%$ y sin información en $9,2 \%$. La situación laboral fue labores domésticas $(39,7 \%)$, trabajo dependiente $(25,1 \%)$, trabajo esporádico $(12,4 \%)$ y trabajo independiente $(7,6 \%)$, con una tasa de inactividad por cesantía u otras razones de $14,9 \%$.

La caracterización socioeconómica de las familias reveló que $8,7 \%$ vivía en situación de indigencia y $31,8 \%$, bajo la línea de la pobreza. Un $39,2 \%$ se ubicaba sobre la pobreza y un $16,4 \%$ poseía un nivel socioeconómico medio. No se consignó esta información para un 3,9\% de las familias.

Debido a la importancia de tener datos lo más completos posibles para realizar los análisis, se seleccionaron los casos que tuvieran menos de $10 \%$ de respuestas omitidas en el instrumento NCFAS y contaran con el $100 \%$ de puntuaciones en los ítems globales de las cinco dimensiones. Usando estos criterios, se identificó una muestra de 528 casos $(89,3 \%$ de la muestra original, $N$ =591). Al tratarse de una muestra no probabilística, se estudiaron posibles diferencias entre el grupo de estudio $(N=528)$ y el grupo excluido $(N=63)$ en variables sociodemográficas y de salud mental. Se realizaron pruebas de $\chi^{2}$ y ANOVA (dependiendo del nivel de medición de cada variable) para determinar diferencias entre los grupos, no encontrándose diferencias estadísticamente significativas para ninguna de las siguientes variables: edad del niño, $F(1,586)$ $=0,498, p=0,481$; edad del cuidador, $F(1$, $336)=0,224, p=0,63$; sexo del niño, $\chi^{2}(1, N$ $=591)=1,1, p=0,292$; sexo del cuidador, $\chi^{2}(1, N=591)=0,963, p=0,327$; escolaridad del niño, $F(1,551)=0,27, p=0,869$; escolaridad del cuidador, $F(1,320)=1,58, p$
= 208; diagnóstico DSM-IV del niño, $\chi^{2}(1, N$ $=591)=0,054, p=0,817)$; diagnóstico DSMIV del cuidador, $\chi^{2}(1, N=591)=4,067, p=$ 0,044; tipo de estructura familiar, $\chi^{2}(1, N=$ $591)=15,753, p=0,072$; y situación económica, $\chi^{2}(1, N=591)=6,1, p=0,408$.

\section{Procedimiento}

Los equipos profesionales recibieron una capacitación de dos días de duración, entrenándose en el uso de la escala sobre la base de los materiales de capacitación aportados por NFPN y sobre contenidos de la Protectora de la Infancia. Luego, aplicaron la escala a los niños y sus familias en un proceso de tres etapas: a) ingreso del caso; b) recopilación de información; y c) puntuación de la NCFAS, como se detalla a continuación.

El proceso comenzó en diciembre de 2007 con la postulación o fase de ingreso, en la que se completó una ficha electrónica de postulación que permitió definir la pertinencia del caso, según criterios predefinidos de focalización en familias con alto riesgo de maltrato y negligencia infantil. El tiempo estimado fue una a dos semanas.

Una vez seleccionados los casos, comenzó la fase de recopilación de información, contando los equipos profesionales con criterios y orientaciones técnicas para indagar sistemáticamente la información requerida al completar la NCFAS. Las fuentes de información usadas fueron: a) informes de las redes de derivación, b) pauta de observación en visita domiciliaria, c) pautas para entrevistar a los actores significativos, d) ecomapa de redes formales e informales y e) diagrama familiar.

Con toda esta información disponible, los profesionales y técnicos realizaron reuniones clínicas para completar la NCFAS en el primer punto de medición (Ingreso), traspasando esta información a una ficha electrónica. El tiempo estimado fue de uno a dos meses para cada caso.

Para controlar la rigurosidad del procedimiento de evaluación, el Director/a de cada programa monitoreó y verificó la recopilación de la información requerida y los criterios usados para establecer las puntuaciones. La recolección de datos y fichas 
electrónicas se realizó entre abril y julio de 2008.

Un tema importante fue el cuidado y resguardo de los aspectos éticos. Si bien la recolección de información sensible para fines diagnósticos es requisito para participar en el programa, se preparó un formulario de consentimiento informado a ser firmado por el/los cuidador/es, que consignó la confidencialidad en términos de anonimato y autorizó el uso de los registros con fines de evaluación e investigación. Además, se tomó especial cautela para asegurar la confidencialidad y anonimato de los registros, tanto físicos como digitales, y en el análisis y comunicación de resultados.

\section{Análisis de Datos}

Previo a evaluar la consistencia interna y dimensionalidad del instrumento, se realizaron análisis descriptivos para tener evidencia de la forma de la distribución de las variables estudiadas. Esto es de especial cuidado porque el nivel de medición de las variables de la NCFAS es ordinal -el mínimo necesario para examinar dimensionalidad usando análisis factorial exploratorio-, interesando no vulnerar el supuesto de normalidad, según lo planteado por Fabrigar, Wegener, MacCallum y Strahan (1999) y Tabachnik y Fidell (1996). Los principales estadísticos descriptivos de los ítems de la NCFAS (media, desviación estándar, asimetría y curtosis) son presentados en primer lugar.

Para el análisis de la consistencia interna de los indicadores que componen las dimensiones de la NCFAS se utilizó el coeficiente alfa de Cronbach. Se consideraron los 31 ítems específicos, excluyendo los ítems de ponderación global, considerando que son dependientes del resto de los ítems específicos (se puntúan después de calificar todos los ítems específicos en cada dimensión).

Para explorar la validez de constructo se realizó un análisis factorial exploratorio. Al igual que en el análisis anterior, se excluyeron los ítems de evaluación global de cada escala. El índice de adecuación muestral Kaiser-Meyer-Olkin $(0,86)$ y la prue- ba de esfericidad de Bartlett $\left(\chi^{2}[465, N=\right.$ $528]=4814,73, p=0,000)$ indican que la matriz de correlaciones entre los 31 ítems seleccionados es apropiada para realizar un análisis factorial exploratorio. Se empleó como método de estimación la factorización de ejes principales (principal axis factoring), al ser altamente recomendado en la literatura (Fabrigar et al., 1999). Los análisis fueron replicados utilizando otros métodos de estimación, como máxima verosimilitud (maximum likelihood) y mínimos cuadrados no ponderados (unweighted least square), obteniendo resultados similares.

El número de factores de la solución se determinó considerando la regla de KaiserGutmann (K-G) junto con el scree test de Catell (Tabachnick \& Fidell, 1996), arrojando un rango entre uno y ocho factores. Si bien estos son métodos ampliamente usados, han sido criticados por sobreestimar o subestimar el número de factores, entre otros aspectos (Henson \& Roberts, 2006; Hoyle \& Duvall, 2004; Preacher \& MacCallum, 2003). Por lo anterior, se consideró como criterio adicional el Parallel Analysis (PA) (Hayton, Allen \& Scarpello, 2004; Horn, 1965, citado en Preacher \& MacCallum, 2003) y el Minimum Average Partial (MAP) (Zwick \& Vellicer, 1986, citado en Henson \& Roberts, 2006). Considerando estos criterios en su conjunto, más la interpretabilidad de las soluciones encontradas, se decidió por una solución de cinco factores.

Para permitir una interpretación simple de la solución, se realizó la rotación ortogonal de la matriz factorial utilizando el método Equamax Normalizado. Para interpretar la estructura de los factores se consideraron cargas factoriales iguales o superiores a 0,35 en la matriz factorial rotada (Hogarty, Hines, Kromrey, Ferron \& Mumford, 2005).

\section{Resultados}

\section{Estadisticos Descriptivos}

En la Tabla 3 se presentan estadísticos descriptivos de las variables de la NCFAS analizadas. Puede observarse que los puntajes promedio de los dominios variaron 
Tabla 3

Características de la Distribución de las Puntuaciones al Ingreso de los Ítems Globales de la NCFAS

\begin{tabular}{lcccccc}
\hline Ítem Global & Promedio & Mediana & Moda & $D E$ & Asimetría & Curtosis \\
\hline Entorno & $-1,02$ & -1 & -1 & 0,94 & 0,265 & 0,140 \\
Competencias Parentales & $-1,27$ & -1 & -1 & 0,89 & 0,201 & 0,268 \\
Interacciones Familiares & $-1,22$ & -1 & -1 & 0,87 & 0,361 & $-0,020$ \\
Seguridad Familiar & $-1,19$ & -1 & -1 & 0,88 & 0,346 & 1,234 \\
Bienestar del Niño & $-0,96$ & -1 & -1 & 0,81 & 0,083 & $-0,133$ \\
\hline
\end{tabular}

$N=528$

entre - 1,27 y -0,96, con desviaciones estándar entre 0,81 y 0,94 . La mediana fue - 1 en todas las dimensiones, valor muy similar al promedio. El rango de puntuación varió entre -3 y +2 en cada dimensión, coincidiendo con el rango teórico posible. En cuanto a la distribución, no se observaron valores extremos de asimetría y curtosis, salvo en el ítem Seguridad Familiar, que fue ligeramente superior a 1. De acuerdo a Tabachnik y Fidell (1996) y Fabrigar et al. (1999), estos resultados sugieren que las puntuaciones de la NCFAS no tendrían problemas de distribución, lo que permite efectuar el análisis factorial sin requerir transformaciones de las variables. Que los promedios de las dimensiones se inclinen hacia el rango problemático de la escala (-1) se explica porque las familias presentan mayor cantidad de problemas que fortalezas al ingresar al programa, dada su focalización en situaciones de maltrato infantil y negligencia de moderada gravedad.

\section{Consistencia Interna}

Entre los criterios propuestos para evaluar la consistencia interna con alfa de Cronbach, Meliá (2000) sugiere como apropiados valores sobre 0,75 , mientras que Streiner y Norman (2003) han señalado que, por convención, sobre 0,7 es un nivel aceptable, sobre 0,8 es alto y sobre 0,9 es muy alto.

Los resultados obtenidos indican que todas las dimensiones de la versión en español de la NCFAS muestran una alta consistencia interna, con valores entre 0,782 (para Seguridad Familiar) y 0,868 (para Entorno). Además, los valores de consistencia interna de todas las dimensiones indican resultados comparables al estudio de Kirk y Griffith (2007), realizado con la versión original del instrumento y con familias en riesgo de disolución atendidas en programas norteamericanos de preservación familiar (Tabla 4).

Tabla 4

Análisis Comparado de la Consistencia Interna de las Dimensiones de la NCFAS en las Versiones en Español e Inglés

\begin{tabular}{lccc}
\hline Dimensión & $\begin{array}{c}\mathrm{N}^{\circ} \text { de } \\
\text { Ítems }\end{array}$ & $\begin{array}{c}\text { Alfa de Cronbach } \\
\text { Versión en Español } \\
(N=528)\end{array}$ & $\begin{array}{c}\text { Alfa de Cronbach } \\
\text { Versión en Inglés } \\
\left(N=1197^{*}\right)\end{array}$ \\
\hline Entorno & 10 & 0,868 & 0,922 \\
Competencias Parentales & 7 & 0,788 & 0,814 \\
Interacciones Familiares & 5 & 0,796 & 0,771 \\
Seguridad Familiar & 6 & 0,782 & 0,767 \\
Bienestar del Niño & 8 & 0,785 & 0,801 \\
\hline
\end{tabular}

* Estudio de Kirk \& Griffith (2007) en Estados Unidos. 


\section{Validez de Constructo: Solución Factorial}

El modelo de cinco factores explica un $44,3 \%$ de la varianza total de la evaluación familiar. Al interpretar la matriz factorial rotada, llama la atención en la Tabla 5 que las cargas de los ítems dentro de los factores son relativamente homogéneas entre sí para todos los factores de la solución, no observándose ítems excesivamente dominantes. $\mathrm{Al}$ mismo tiempo, la mayoría de los ítems cargan en un solo factor, lo que favorece una estructura simple. Las excepciones son dos ítems que cargan en dos factores a la vez y cinco ítems que tienen cargas factoriales inferiores al criterio establecido. De estos últimos, tres tienen cargas bajo 0,2 y uno presenta una carga de 0,325 (estos últimos no aparecen reflejados en la Tabla 6).

Los factores del modelo propuesto son: 1) Entorno, 2) Competencias Parentales, 3) Protección Familiar, 4) Bienestar del Niño y 5) Bienestar del Cuidador. La composición de cada uno de estos factores se señala a continuación (comparar Tablas 1 y 6 ).

El Factor 1 fue nombrado Entorno e incluye ocho de los nueve ítems específicos de la dimensión original de la NCFAS: ingreso/empleo, manejo financiero, comida y nutrición, higiene personal, habitabilidad de la vivienda, entorno de aprendizaje, estabilidad de la vivienda y seguridad de la comunidad, manteniéndose dicha dimensión prácticamente idéntica a la versión original de la escala.
El Factor 2 fue nombrado Competencias Parentales y reúne seis ítems. Este factor retiene tres de los seis ítems originales: supervisión del niño, entrega de oportunidades de desarrollo/aprendizaje y prácticas disciplinarias. Además, integra los ítems apego con el niño y expectativas sobre el niño (de Interacciones Familiares) y el ítem ausencia/presencia de negligencia en el niño (de Seguridad Familiar). El ítem entorno de aprendizaje está incorporado tanto en este factor como en el Factor 1. Desde la perspectiva de los autores, resulta interesante considerar en futuros estudios su pertenencia al factor Competencias Parentales, puesto que la puntuación +2 "se refiere al entusiasmo de los cuidadores por enseñar a los niños. La familia tiene rutinas para el juego y el estudio. El tiempo está planificado para la lectura, ir a excursiones y actividades estructuradas..." (Kirk et al., 2007, p. 14). Además, cabe destacar que este ítem muestra una carga levemente mayor en este factor $(0,482)$ que en el factor Entorno $(0,465)$.

El Factor 3 fue nombrado Protección $\mathrm{Fa}$ miliar e incluye cinco ítems. Está compuesto por dos de los cinco ítems de la dimensión original de la NCFAS: ausencia/presencia de violencia doméstica entre los progenitores/cuidadores y ausencia/presencia de abuso emocional en el niño. Asimismo, incorpora los ítems relación entre los progenitores/cuidadores y apoyo mutuo dentro

Tabla 5

Porcentaje de Varianza Explicada por la Solución Factorial de la Versión en Español de la NCFAS

\begin{tabular}{|c|c|c|c|c|c|c|}
\hline \multirow[b]{2}{*}{ Factor } & \multicolumn{3}{|c|}{ Inicial } & \multicolumn{3}{|c|}{ Final } \\
\hline & $\mathrm{EV}$ & $\%$ Varianza & $\begin{array}{c}\% \\
\text { Acumulado }\end{array}$ & $\mathrm{EV}$ & $\%$ Varianza & $\begin{array}{c}\% \\
\text { Acumulado }\end{array}$ \\
\hline 1 & 7,93 & 25,56 & 25,56 & 3,45 & 11,12 & 11,12 \\
\hline 2 & 2,98 & 9,61 & 35,18 & 3,31 & 10,68 & 21,80 \\
\hline 3 & 2,56 & 8,26 & 43,44 & 2,80 & 9,04 & 30,84 \\
\hline 4 & 1,47 & 4,74 & 48,18 & 2,51 & 8,09 & 38,93 \\
\hline 5 & 1,42 & 4,57 & 52,74 & 1,67 & 5,38 & 44,31 \\
\hline 6 & 1,34 & 4,32 & 57,06 & & & \\
\hline 7 & 1,17 & 3,60 & 60,66 & & & \\
\hline 8 & 1,00 & 3,24 & 63,90 & & & \\
\hline
\end{tabular}


Tabla 6

Matriz Factorial Rotada para los Ítems de la Versión en Español de la NCFAS

\begin{tabular}{|c|c|c|c|c|c|}
\hline Ítem & $\begin{array}{c}\mathrm{F} 1 \\
\text { Entorno }\end{array}$ & $\begin{array}{c}\text { F2 } \\
\text { Competencias } \\
\text { Parentales }\end{array}$ & $\begin{array}{c}\text { F3 } \\
\text { Protección } \\
\text { Familiar }\end{array}$ & $\begin{array}{c}\text { F4 } \\
\text { Bienestar del } \\
\text { Niño }\end{array}$ & $\begin{array}{c}\text { F5 } \\
\text { Bienestar del } \\
\text { Cuidador }\end{array}$ \\
\hline Ingreso-empleo & 0,723 & & & & \\
\hline Manejo financiero & 0,694 & & & & \\
\hline Comida y nutrición & 0,693 & & & & \\
\hline Higiene personal & 0,620 & & & & \\
\hline Habitabilidad de la vivienda & 0,615 & & & & \\
\hline Entorno de aprendizaje* & 0,465 & 0,482 & & & \\
\hline Estabilidad de la vivienda & 0,457 & & & & \\
\hline Seguridad en la comunidad & 0,370 & & & & \\
\hline Supervisión del niño & & 0,676 & & & \\
\hline Apego con el niño & & 0,643 & & & \\
\hline Presencia de negligencia en el niño & & 0,626 & & & \\
\hline $\begin{array}{l}\text { Entrega de oportunidades de } \\
\text { desarrollo }\end{array}$ & & 0,613 & & & \\
\hline Prácticas disciplinarias & & 0,584 & & & \\
\hline Expectativas sobre el niño & & 0,516 & & & \\
\hline Relación entre los cuidadores & & & 0,791 & & \\
\hline $\begin{array}{l}\text { Presencia de violencia doméstica } \\
\text { entre cuidadores }\end{array}$ & & & 0,783 & & \\
\hline $\begin{array}{l}\text { Presencia de abuso emocional en } \\
\text { el niño }\end{array}$ & & & 0,522 & & \\
\hline Apoyo mutuo dentro de la familia & & & 0,450 & & \\
\hline $\begin{array}{l}\text { Uso de drogas-alcohol de los } \\
\text { cuidadores }\end{array}$ & & & 0,368 & & \\
\hline Comportamiento del niño & & & & 0,800 & \\
\hline Desempeño escolar & & & & 0,585 & \\
\hline $\begin{array}{l}\text { Relación con el progenitor- } \\
\text { cuidador }\end{array}$ & & & & 0,530 & \\
\hline Relación con pares & & & & 0,522 & \\
\hline Relación con hermanos & & & & 0,470 & \\
\hline Salud física de los cuidadores & & & & & 0,480 \\
\hline Transporte & & & & & 0,451 \\
\hline Salud mental de los cuidadores* & & & 0,471 & & 0,436 \\
\hline $\begin{array}{l}\text { Presencia de abuso físico en el } \\
\text { niño** }\end{array}$ & & & 0,325 & & \\
\hline \multicolumn{6}{|l|}{$\begin{array}{l}\text { Presencia de abuso sexual en el } \\
\text { niño*** }\end{array}$} \\
\hline Salud mental del niño*** & & & & & \\
\hline $\begin{array}{l}\text { Cooperación a permanecer con la } \\
\text { familia*** }\end{array}$ & & & & & \\
\hline
\end{tabular}

* El ítem carga en dos factores.

** $\quad$ El ítem muestra una carga factorial menor a 0,35.

*** El ítem muestra una carga factorial inferior a 0,2 en todos los factores. 
de la familia (dimensión Interacciones $F a$ miliares). El ítem uso de drogas/alcohol del progenitor(es)/cuidador(es) es el último asociado y tiene una carga factorial en el límite de la regla establecida $(0,37)$. El ítem salud mental de los progenitores/cuidadores está presente en dos factores (3 y 5), pero se ha optado por conservarlo en el Factor 5 (Bienestar del Cuidador) por razones de congruencia teórico-práctica: dado que otro de los ítems del Factor 5 es salud física, se facilita para el operador visualizar al cuidador en ambos aspectos de su salud (física y mental) de forma integral y no parcelada.

El Factor 4 fue nombrado Bienestar del Niño y reúne cinco de los siete ítems específicos de la dimensión original: comportamiento del niño, desempeño escolar, relación con el progenitor/cuidador, relación con pares y relación con hermanos, quedando fuera los ítems salud mental del niño y cooperación/motivación a permanecer en la familia.

Finalmente, el Factor 5 fue llamado Bienestar del Cuidador e incluye los ítems salud física del progenitor/cuidador y salud mental del progenitor/cuidador de la dimensión original Competencias Parentales. Además, se observa que el ítem transporte (de la dimensión original Entorno) carga en este factor; esto resulta coherente al revisar la definición correspondiente a la puntuación -3: "se refiere a una familia que no tiene transporte disponible, lo que en cambio, inhibe el trabajo, incrementa el aislamiento social, y/o limita el acceso a servicios" (Kirk et al., 2007, p. 13). Esta definición da cuenta del grado de accesibilidad/aislamiento frente a los distintos servicios locales (salud, educación, municipales). Se hipotetiza que esto podría relacionarse con el bienestar físico y mental de los cuidadores a través de las limitaciones que el aislamiento genera, especialmente en adultos con extensas jornadas laborales o que deben realizar traslados que superan sus recursos y/o capacidad de movilización disponible. La falta de acceso a estos servicios, a su vez, podría impactar sus condiciones de salud mental, salud física y bienestar en general; de ahí que resulte teóricamente coherente la asociación estadística de este ítem con otros ítems ligados al bienestar del cuidador.
Discusión y Conclusiones

Este estudio fue desarrollado con un tamaño muestral adecuado para sus objetivos, el proceso de recolección de datos se hizo de manera confiable y consistente, la capacitación a los profesionales siguió estándares comparables a los estudios iniciales de la NCFAS y el contexto de aplicación fue similar, lo que aumenta su validez ecológica.

Los análisis estadísticos dan cuenta de adecuadas propiedades psicométricas y una alta consistencia interna de la escala. Estos hallazgos son consistentes con lo reportado en investigaciones previas sobre la NCFAS (Kirk et al., 2005; Kirk \& Griffith, 2007; Reed, 1998; Reed-Ashcraft et al., 2001). El estudio aporta evidencia de una estructura dimensional que resulta comparable a la de la escala original, si bien se presentan algunas diferencias. Los elementos comunes tienen relación con el número de factoresdimensiones (cinco) y con la estructura de dos factores respecto a las cinco dimensiones originales (Factor 1, Entorno, y Factor 4, Bienestar del Niño). Otros dos factores (Factores 2 y 3 ) representan una combinación de ítems conceptualmente muy relacionados, pero donde hay dominancia de una de las dimensiones originales (Competencias Parentales y Seguridad Familiar, respectivamente). La dimensión original Interacciones Familiares no se sostiene inicialmente tras el análisis factorial, distribuyéndose sus ítems entre los factores Competencias Parentales (Factor 2) y Protección Familiar (Factor 3).

Al estudiar la nueva organización de ítems en la dimensión Competencias $P a$ rentales, se observa que el análisis factorial deja fuera los ítems de la versión original de la NCFAS relativos al estado funcional del cuidador (salud física/mental y consumo de drogas/alcohol) e incorpora ítems relativos al cuidado (supervisión, negligencia), estimulación (oportunidades de desarrollo, entorno de aprendizaje), formación (prácticas disciplinarias) y la relación (expectativas, apego) con el niño, niña o adolescente, lo que resulta plenamente coherente desde el marco teórico actual sobre competencias parentales (Barudy \& Dantagnan, 2005). 
Si bien los ítems del factor Protección Familiar apuntan a elementos que pueden definir una situación de desprotección infantil (violencia intrafamiliar, maltrato emocional, abuso de drogas/alcohol), llama la atención el hallazgo inesperado de que el ítem abuso físico en el niño no haya cargado en el factor con la fuerza suficiente como para incluirse $(0,325)$. Esto podría deberse a la dificultad de evaluar maltrato infantil, dificultad que se ha ligado a la falta de conceptualización y de criterios claros y precisos para definir qué es maltrato y qué no (Haz \& Ramírez, 2002). Siendo un elemento crítico de evaluar en casos de desprotección infantil (Arruabarrena, 2009), una tarea prioritaria para futuras adaptaciones sería perfeccionar las definiciones operacionales de la escala y la capacitación y consenso entre los operadores que califican estas situaciones. El mismo aspecto se repite en el ítem abuso sexual en el niño, pudiendo obedecer a idénticas razones.

El ítem salud mental del niño no cargó en el factor Bienestar del Niño, pudiendo responder a falta de especialización de los operadores en diagnóstico infanto-juvenil, lo que se explicaría por tratarse de un programa de apoyo familiar integral y no de un servicio de salud mental infantil. Tal vez sería necesario complementar la evaluación con otros instrumentos, como cuestionarios de problemas clínicos infanto-juveniles, para mejorar este aspecto. Por otra parte, el ítem cooperación a permanecer con la familia puede presentar sesgos culturales; las familias latinoamericanas tienden a ser más aglutinadas que las angloparlantes $\mathrm{y}$, por tanto, puede ser más difícil que un niño, aun cuando esté viviendo situaciones dolorosas de maltrato y desprotección, manifieste interés por desligarse de su familia. Sin embargo, esta hipótesis requeriría de un estudio cualitativo que indague en la percepción de los propios niños/as y adolescentes al respecto.

Un elemento interesante a considerar es que el análisis factorial exploratorio sugiere la configuración de una nueva dimensión, denominada tentativamente Bienestar del Cuidador (Factor 5) y que agrupa condiciones de salud física y mental de las figuras parentales y su acceso o aislamiento de los servicios locales en diversas áreas impor- tantes para una adecuada calidad de vida, como son la salud, educación y servicios municipales. Tanto desde un punto de vista estadístico como teórico, la nueva estructura factorial muestra coherencia y aplicabilidad práctica, por lo que podría considerarse como un punto de partida para realizar futuras adaptaciones locales de un instrumento de evaluación familiar integral.

De acuerdo a la experiencia práctica de la Protectora de la Infancia en sus programas PIB Viviendo en Familia, la NCFAS es un instrumento de evaluación integral de la familia que resulta pertinente y de utilidad práctica, por cuanto permite capturar componentes ecológicos y sistémicos de su funcionamiento, replicando así la experiencia internacional al respecto (Fernandez, 2007). Así, esta escala da cuenta de dimensiones relevantes para el diagnóstico y evaluación en profundidad de un amplio abanico de situaciones psicosociales, con un mínimo de capacitación a los evaluadores y usando una cantidad acotada de tiempo de administración. Sin embargo, es necesario precisar que si bien toma poco tiempo el registro propiamente tal, se requiere una inversión considerable de tiempo y recursos en la etapa previa de levantamiento de la información, necesaria para realizar una adecuada ponderación de cada dimensión.

El gran aporte práctico de este instrumento va en dos direcciones: por un lado, permite organizar la información que habitualmente recopilan los programas sociales (por ejemplo, informes de redes de derivación, entrevistas y visitas domiciliarias), para orientar la toma de decisiones y la planificación de las intervenciones; por otra parte, permite evaluar en forma precisa (con un diseño pre-post) los resultados obtenidos en la intervención en dimensiones relevantes al quehacer de los programas (Coll \& Stewart, 2006; De la Rosa et al., 2009; Fernandez, 2007; Kirk \& Griffith, 2007; Richardson, 2008). Si bien resulta necesario desarrollar estudios que aborden la adaptación y estandarización del instrumento para su uso en la población local, el presente estudio entrega evidencia que respalda el empleo de la versión en español de la NCFAS como una herramienta de evaluación y diagnóstico multidimensional de la familia en este tipo de programas. 


\section{Referencias}

Appleyard, K., Egeland, B., Dulmen, M. H. M. \& Sroufe, L. A. (2005). When more is not better: The role of cumulative risk in child behavior outcomes. Journal of Child Psychology and Psychiatry, 46, 235-245.

Arruabarrena, M. I. (2009). Procedimiento y criterios para la evaluación y la intervención con familias y menores en el ámbito de la protección infantil. Papeles del Psicólogo, 30, 13-23.

Barudy, J. \& Dantagnan, M. (2005). Los buenos tratos a la infancia. Parentalidad, apego y resiliencia. Barcelona: Gedisa.

Belsky, J. (1993). Etiology of child maltreatment: A developmental-ecological analysis. Psychological Bulletin, 114, 314-334.

Bronfenbrenner, U. (1986). Ecology of the family as a context for human development: Research perspectives. Developmental Psychology, 22, 723742.

Bronfenbrenner, U. (1987). La ecología del desarrollo humano. Barcelona: Paidós.

Carretero-Dios, H. \& Pérez, C. (2007). Standards for the development and review of instrumental studies: Considerations about test selection in psychological research. International Journal of Clinical and Health Psychology, 7, 863-882.

Coll, K. \& Stewart, R. (2006). Ada County Family Violence Court Grant Project: Comprehensive evaluation report. Phoenix, AZ: Rocky Mountain Quality Improvement Center.

Connell-Carrick, K. (2003). A critical review of the empirical literature: Identifying correlates of child neglect. Child and Adolescent Social Work Journal, 20, 389-425.

De la Rosa, I. A., Perry, J., Dalton, L. E. \& Johnson, V. (2005). Strengthening families with first-born children: Exploratory story of the outcomes of a home visiting intervention. Research on Social Work Practice, 15, 323-338.

De la Rosa, I. A., Perry, J. \& Johnson, V. (2009). Benefits of increased home-visitation services: Exploring a case management model. Family \& Community Health, 32, 58-75.

Fabrigar, L. R., Wegener, D. T., MacCallum, R. C. \& Strahan, E. J. (1999). Evaluating the use of exploratory factor analysis in psychological research. Psychological Methods, 4, 272-299.

Fernandez, E. (2007). Supporting children and responding to their families: Capturing the evidence on family support. Children and Youth Services Review, 29, 1368-1394.

Gómez, E. \& Haz, A. M. (2008). Intervención familiar preventiva en programas colaboradores del SENAME: la perspectiva del profesional. Psykhe, 17(2), 53-65.

Gómez, E., Muñoz, M. \& Haz, A. M. (2007). Familias multiproblemáticas y en riesgo social: características e intervención. Psykhe, 16(2), 4354.

Hayton, J. C., Allen, D. G. \& Scarpello, V. (2004). Factor retention decisions in exploratory factor analysis: A tutorial on parallel analysis. Organizational Research Methods, 7, 191-205.
Haz, A. M. \& Ramírez, V. (2002). Adaptación del Child Abuse Potential Inventory en Chile: análisis de las dificultades y desafíos de su aplicación a partir de dos estudios chilenos. Child Abuse \& Neglect, $26,481-495$.

Henson, R. K. \& Roberts, J. K. (2006). Use of exploratory factor analysis in published research. Educational and Psychological Measurement, 66, 393-416.

Herrenkohl, T. I. \& Herrenkohl, R. C. (2007). Examining the overlap and prediction of multiple forms of child maltreatment, stressors, and socioeconomic status: A longitudinal analysis of youth outcomes. Journal of Family Violence, 22, 553-562.

Hogarty, K. Y., Hines, C. V., Kromrey, J. D., Ferron, J. M. \& Mumford, K. R. (2005). The quality of factor solutions in exploratory factor analysis: The influence of sample size, communality, and overdetermination. Educational and Psychological Measurement, 65, 202-226.

Hoyle, R. H. \& Duvall, J. L. (2004). Determining the number of factors in exploratory and confirmatory factor analysis. En D. Kaplan (Ed.), Handbook of quantitative methodology for the social sciences (pp. 301-315). Thousand Oaks, CA: Sage Publications.

Johnson, M. A., Stone, S., Lou, C., Vu, C., Ling, J., Mizrahi, P. \& Austin, M. J. (2006). Family assessment in child welfare services: Instrument comparisons. Berkeley, CA: University of California, Center for Social Services Research.

Kirk, R. S. \& Griffith, D. P. (2007). An examination of intensive family preservation services. Buhl, ID: The Anne Casey Foundation. Extraído el 20 enero, 2009, de http://www.nfpn.org/articlesmainmenu-34/105-ifps-research-report.html

Kirk, R. S., Kim, M. M. \& Griffith. D. P. (2005). Advances in the reliability and validity of the North Carolina Family Assessment Scale. Journal of Human Behavior in the Social Environment, 11(3/4), 157-176.

Kirk, R. S., Reed-Ashcraft, K. B \& National Family Preservation Network (2007). Escala de Evaluación Familiar de Carolina del Norte. Buhl, ID: National Family Preservation Network.

Larraín, S. \& Bascuñán, C. (2008). Maltrato infantil $y$ relaciones familiares en Chile. Análisis comparativo 1994 - 2000 - 2006. Santiago, Chile: Fondo de las Naciones Unidas para la Infancia.

Larraín, M. E., Zegers, B., Diez, I., Trapp, A. \& Polaino-Lorente, A. (2003). Validez y confiabilidad de la versión española de la Escala del Estilo de Funcionamiento Familiar (EFF) de Dunst, Trivette \& Deal para el diagnóstico del funcionamiento familiar en la población chilena. Psykhe, 12(1), 195-211.

Matus, T. (2007). El peso que queda: condiciones de efectividad en los programas de intervención social. Perspectivas CEES-UC, 3, 5-17. Extraído el 21 enero, 2009, de http://www.cees.cl

Meliá, J. L. (2000). Teoría de la fiabilidad y la validez. Valencia: Cristóbal Serrano.

National Family Preservation Network (2009). Overview of assessment tools. Buhl, ID: Autor. Extraído el 25 julio, 2009, de http://www.nfpn.org/images/stories/ files/assessment_tools_overview.pdf 
Preacher, K. J. \& MacCallum, R. C. (2003). Repairing Tom swift's electric factor analysis machine. Understanding Statistics, 2, 13-43.

Reed, K. B. (1998). The reliability and validity of the North Carolina Family Assessment Scale. Chapel Hill, NC: The University of North Carolina at Chapel Hill.

Reed-Ashcraft, K., Kirk, R. S. \& Fraser, M. W. (2001). The reliability and validity of the North Carolina Family Assessment Scale. Research on Social Work Practice, 11, 503-520.

Richardson, B. (2008). Comparative analysis of two community-based efforts designed to impact disproportionality. Child Welfare Journal, 87, 297-317.

Rodríguez, D. \& Ríos, R. (2007). Las organizaciones sociales en una sociedad compleja. Perspectivas CEES-UC, 2, 5-27. Extraído el 21 enero, 2009, de http://www.cees.cl

Fecha de recepción: Julio de 2009.

Fecha de aceptación: Enero de 2010.
Santana-Tavira, R., Sánchez-Ahedo, R. \& HerreraBasto, E. (1998). El maltrato infantil: un problema mundial. Salud Pública de México, 40, 1-8.

Stockhammer, T. F., Salzinger, S., Feldman, R. S., Mojica, E. \& Primavera, L. H. (2001). Assessment of the effect of physical child abuse within an ecological framework: Measurement issues. Journal of Community Psychology, 29, 319-244.

Streiner, D. \& Norman, G. (2003). Health measurement scales: A practical guide to their development and use ( $2^{\text {nd }}$ ed.). New York: Oxford University Press.

Tabachnick, B. G. \& Fidell, L. S. (1996). Principal components and factor analysis. En B. G. Tabachnick \& L. S. Fidell, Using multivariate statistics ( $3^{\text {rd }}$ ed., pp. 635-707). New York: HarperCollins.

Walker, R., Minor-Schork, D., Bloch, R. \& Esinhart, J. (1996). High risk factors for rehospitalization within six months. Psychiatric Quarterly, 67, 235243. 